\title{
Validação da escala de motivação no esporte (SMS) no futebol para a língua portuguesa brasileira
}

CDD. 20.ed. 796.011

796.33

\author{
Varley Teoldo da COSTA ${ }^{* * *}$ \\ Maicon Rodrigues ALBUQUERQUE ${ }^{* * \alpha}$ \\ Mariana Calábria LOPES ${ }^{* * * *}$ \\ Franco NOCE****** \\ Israel Teoldo da COSTA ${ }^{* * * * * *}$ \\ Renato Melo FERREIRA**** \\ Dietmar Martin SAMULSKI*
}

*Escola de Educação Física, Fisioterapia e Terapia Ocupacional, Universidade Federal de Minas Gerais.

**Centro Universitário de Belo Horizonte.

***Faculdade de Ciências Humanas, Sociais e da Saúde, Instituto

Superior de Educação Anísio Teixeira.

****Universidade de Heidelberg - Alemanha. *****Universidade $\mathrm{Fe}$ deral do Ouro Preto.

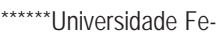
deral de Viçosa.

0 objetivo deste estudo foi submeter o questionário Sport Motivation Scale (SMS) ao processo de validação transcultural da Escala de Motivação no Esporte para a língua portuguesa brasileira (EME-BR), verificando sua validade e aplicabilidade na população de jovens atletas brasileiros de futebol de alto nível. Participaram deste estudo 370 atletas. Utilizou-se o Instrumento denominado Escala de Motivação no Esporte - versão brasileira (EME-BR), no qual é composto por 28 itens, dividido em sete dimensões. Para a tradução e adaptação do instrumento, foram adotados os procedimentos sugeridos na literatura. Os resultados da análise fatorial confirmatória, demonstraram que com exceção do teste $S-B x^{2}$ que foi significante $(p<0,001)$, todas as outras medidas de ajuste absoluto (GFI e RMSEA) e de ajuste incremental (NFI, NNFI e AGFI), apresentaram valores dentro dos aceitáveis pela literatura. No que diz respeito ao termo de confiabilidade interna, pode-se observar que somente duas dimensões atingiram o valor mínimo $(>0,70)$, entretanto, as demais dimensões, aproximam-se do índice desejável. Contudo, os resultados obtidos no presente estudo dão algum suporte para a validade da EME-BR com jogadores brasileiros jovens de futebol de alto nível.

UnItermos: Motivação; Futebol de alto nível; Validação.

\section{Introdução}

Atualmente as áreas da Psicologia Esportiva e da Educação Física têm aumentado o seu interesse no processo de validação de instrumentos psicométricos que visam medir um determinado constructo psicológico. FONSECA (2001) realizou uma meta-análise no Sport Discus do Sport Information Resource Center (SIRC) sobre as temáticas de publicação na área da Psicologia do Esporte e identificou 1888 artigos publicados sobre o tema motivação entre os anos de 1991 a 2000, classificando assim, a temática em primeiro lugar como área de interesse para a investigação por parte dos pesquisadores. $\mathrm{O}$ mesmo autor menciona também neste estudo, que a área mais publicada é a de motivação (15,7\%). Corroborando com os dados acima, RuIZ, BENEDiCTO e Dosil (2004), analisaram a produção científica de uma revista no idioma espanhol entre os anos de 1995 a 2003 também destacam a motivação $(19,85 \%)$ como a principal temática investigada na Psicologia Esportiva. Observa-se com base nestes estudos, que tanto na língua inglesa como espanhola existe uma atratividade dos pesquisadores pela área.

$\mathrm{Na}$ literatura é possível encontrar inúmeras definições sobre o conceito da motivação, sendo que uma definição clássica é a de SAMULSKI (1995), que conceitua a motivação esportiva como um processo ativo, intencional e dirigido a uma meta, o qual depende da interação dos fatores pessoais e ambientais (ver Samulski, 2009). Dosil (2008) lembra que esta é uma variável imprescindível para o sucesso esportivo de um atleta ou equipe, na medida em que reflete diretamente o processo de aprendizagem 
de uma modalidade, a persistência em continuar praticando a mesma e a busca pela melhoria diária no desempenho (Albuquerque, Costa, Samulski $\&$ Noce, 2008). A motivação pode, também, ser conceituada como um modelo hipotético usado para descrever as forças internas (motivação intrínseca) e/ou externas (motivação extrínseca) do indivíduo que estão em constante processo de simbiose com o meio ambiente, gerando assim, comportamentos motivantes ou desmotivantes (VALLERAND, 2007).

O presente estudo tem como principal referencial a Teoria de Autodeterminação (TAD), proposta por DeCI e Ryan $(1985,2000)$ onde o comportamento humano é incentivado por três necessidades: autonomia, competência e relação social, que parecem ser essenciais para o desenvolvimento social e o bemestar pessoal do indivíduo (DECI \& RYAN, 2000; Ryan \& Deci, 2000). A autonomia pode ser definida como autodeterminação do indivíduo e compreende a necessidade do ser humano de ser agente de suas ações, consequentemente determinando o seu próprio comportamento. Já a competência está relacionada à necessidade de produzir resultados comportamentais. Por fim, a relação social refere-se à necessidade das pessoas de terem interaçôes sociais satisfatórias dentro da sociedade, sendo que esta pode ser caracterizada de duas formas: ser aceito pelo grupo e estar mais próxima de outras pessoas (Deci \& Ryan, 2000; Ryan \& DeCI, 2000).

Além disso, dentro da TAD, DeCI e RYAN (1985) propõe uma subteoria denominada Integração dos Organismos (Organismic Integration Theory), que apresenta um modelo multidimensional composto de três tipos de motivação: motivação intrínseca (mais autodeterminada), motivação extrínseca e desmotivação (menos autodetermianda) que estão dispostas em

\section{Métodos}

\section{Amostra}

Por se tratar de uma pesquisa envolvendo menores de idade foi solicitado aos chefes de delegação que assinassem um termo de consentimento autorizando suas equipes e seus respectivos atletas a participarem da pesquisa. Todos os voluntários foram informados sobre os objetivos e os procedimentos que seriam realizados, tendo, em seguida, assinado individualmente um termo de consentimento livre e esclarecido. Este estudo foi aprovado pelo Comitê de pólos distintos dentro de um continuum motivacional (ver DeCI \& Ryan, 2000; Ryan \& DeCI, 2000).

Respaldado pela TAD, Brière, Vallerand, Blais e Pelletier (1995), desenvolveram o instrumento denominado "Échelle de Motivation dans lês Sports" (Escala de Motivação no Esporte) em francês para ser utilizado no contexto esportivo. Posteriormente Pelletier, Tuson, Fortier, Vallerand, Brière e BLAIS (1995), validaram o instrumento para a língua inglesa, denominando-o de SMS (Sport Motivation Scale). Neste estudo eles utilizaram uma amostra de 593 atletas canadenses universitários. Recentemente, o instrumento foi traduzido para outros idiomas como o búlgaro, chinês e grego (ver Pelletier \& SERRAZIN, 2007, p.146).

Tendo como marco inicial da discussão o trabalho de Martens e Webber (2002), atualmente desencadeou-se um importante debate científico sobre as propriedades psicométricas do SMS (ver MALLETT, Kawabata \& Newcombe, 2007; Mallett, Kawabata, Newcombe, Otero-Ferero \& Jackson, 2007; Pelletier, Vallerand \& Sarrazin, 2007). Independente dos próximos embates teóricos parece existir um consenso que o SMS é umas das ferramentas mais utilizadas para avaliar motivação no esporte (PeLLeTIER $\&$ SARRAZIN, 2007) e que todos os ajustes (que vierem a ser ou não confirmados) tem como objetivo tornar o instrumento mais fidedigno e seguro cientificamente.

Portanto, este estudo busca contribuir para esta discussão científica internacional e tem como objetivo principal submeter o questionário Sport Motivation Scale (SMS) ao processo de validação transcultural da Escala de Motivação no Esporte para a língua portuguesa brasileira (EME-BR), verificando sua validade e aplicabilidade na população de jovens atletas brasileiros de futebol de alto nível.
Ética em Pesquisa (COEP) do Centro Universitário de Belo Horizonte - UNI-BH (081/2007).

A amostra foi composta por 370 atletas de futebol do gênero masculino, federados e oriundos das categorias de base infantil, juvenil e juniores dos principais clubes do país, com média de idade de $15,58( \pm 1,19)$ anos, com experiência competitiva média de 5,47 $( \pm 3,11)$ anos.

A escolha intencional desta amostragem deve-se ao fato de terem sido investigados clubes de todas as regiōes do país que possuem atletas nascidos 
em diversas cidades brasileiras. Estas agremiações representam os maiores centros de excelência em formação de atletas de futebol no país e disputam as principais competições nacionais promovidas pela CBF (Confederação Brasileira de Futebol) na categoria de base.

\section{Procedimentos}

A coleta de dados foi realizada nas cidades sedes das competições Copa Brasil de Futebol Infantil e Copa Youth Cup U-15, nas cidades de Votorantim e Belo Horizonte, respectivamente, e contou com o apoio oficial dos organizadores destes dois eventos chancelados pela Confederação Brasileira de Futebol (CBF). No congresso técnico foram definidos os clubes que gostariam de participar da pesquisa e o cronograma de coleta dos dados. Estabelecidas essas metas os pesquisadores se dirigiam aos hotéis e escolas onde as delegações estavam alojadas para a execução da pesquisa, sendo que todos os dados foram coletados antes da estréia das equipes na competição.

\section{Instrumento}

Todos os participantes do estudo responderam a Escala de Motivação no Esporte - versão brasileira (EME-BR), a qual é composta por 28 itens divididos em sete dimensões (QUADRO 1). As questões devem ser respondidas por meio de uma escala Likert de sete pontos que varia de 1 "não corresponde nada" a 7 "corresponde exatamente".

QUADRO 1-Escala de Motivação no Esporte - versão brasileira (EME-BR).

\begin{tabular}{|l|l|l|}
\hline \multicolumn{1}{|c|}{ Nome da dimensão } & \multicolumn{1}{|c|}{ Definição conceitual } & \multicolumn{1}{c|}{ Itens } \\
\hline $\begin{array}{l}\text { "Motivação intrínseca } \\
\text { para conhecer" (MI-C) }\end{array}$ & $\begin{array}{l}\text { Relacionada a fatores pessoais ligados a curiosidade e a busca de com- } \\
\text { preensão que o atleta deseja obter sobre a modalidade praticada. }\end{array}$ & $\begin{array}{l}2,4, \\
23 \text { e } 27\end{array}$ \\
\hline $\begin{array}{l}\text { "Motivação intrínseca para } \\
\text { atingirem objetivos" (MI-AO) }\end{array}$ & $\begin{array}{l}\text { Relacionada a fatores pessoais onde o atleta sente prazer na busca } \\
\text { de novas habilidades e movimentos dentro da modalidade esportiva. }\end{array}$ & $\begin{array}{l}8,12, \\
15 \text { e } 20\end{array}$ \\
\hline $\begin{array}{l}\text { "Motivação intrínseca para } \\
\text { experiências estimulantes" (MI-EE) }\end{array}$ & $\begin{array}{l}\text { Relacionada a fatores pessoais que fazem o atleta buscar experiên- } \\
\text { cias estimulantes no esporte que podem causar excitação, prazer e } \\
\text { divertimento. }\end{array}$ & $\begin{array}{l}1,13, \\
18 \text { e 25 }\end{array}$ \\
\hline $\begin{array}{l}\text { "Motivação extrínseca de } \\
\text { regulação externa" (ME-RE) }\end{array}$ & $\begin{array}{l}\text { Relacionada a fatores ambientais externos ligados a recompensas } \\
\text { oriundas de um bom desempenho, como por exemplo, a conquista } \\
\text { de troféus, recompensas financeiras ou mesmo status perante o } \\
\text { treinador e o grupo }\end{array}$ & $\begin{array}{l}6,16, \\
16\end{array}$ \\
\hline "Motivação extrínseca \\
de introjeção" (ME-I) & $\begin{array}{l}\text { Pressões internas que o atleta pode colocar em si mesmo. Constran- } \\
\text { gimento ou vergonha de estar envolvido em situaçóes onde falham } \\
\text { ou não conseguem o seu melhor desempenho. }\end{array}$ & $\begin{array}{l}9,14 \\
21 \text { e } 26\end{array}$ \\
\hline $\begin{array}{l}\text { "Motivação extrínseca } \\
\text { de identificação" (ME-ID) }\end{array}$ & $\begin{array}{l}\text { Associada aos atletas que participam ativamente de esportes porque } \\
\text { sentem que isto os ajuda a crescer pessoalmente, consideram o es- } \\
\text { porte e a vida em geral. }\end{array}$ & $\begin{array}{l}7,11, \\
17 \text { e } 24\end{array}$ \\
\hline "Desmotivação" (DES) & $\begin{array}{l}\text { Caracteriza-se pelo sentimento de desesperança onde a motivações } \\
\text { extrínsecas ou intrínsecas não afetam o desempenho do atleta que } \\
\text { não sente razão para continuar praticando o esporte. }\end{array}$ & $\begin{array}{l}3,5 \\
19 \text { e } 28\end{array}$ \\
\hline
\end{tabular}

Fonte: Adaptado de PeLLETIER et al. (1995).
Antes da aplicação do EME-BR foi realizado o processo de tradução e adaptação cultural de acordo com as diretrizes estabelecidas por BEATON, Bombardier, Guillemin e Ferraz (2000), as quais podem ser divididas em seis etapas. A primeira etapa foi realizada por dois tradutores independentes que realizaram a tradução original do inglês para o português. $\mathrm{Na}$ segunda etapa esses dois tradutores se reuniram e elaboraram uma primeira versão do instrumento. Esta primeira versão foi submetida na terceira etapa a dois novos tradutores "experts" e independentes, que não tiveram contato com as etapas anteriores, para um processo de retradução do português para o inglês ("backtranslation"). $\mathrm{Na}$ quarta etapa estes instrumentos foram submetidos a um Comitê Avaliador (denominado "Comitte Expert") composto por professores universitários e tradutores juramentados que possuem características de "expertise" para a avaliação de equivalência semântica, idiomática, experimental e conceitual. 
$\mathrm{Na}$ quinta etapa foi realizado um estudo piloto com 30 atletas de uma equipe juvenil de futebol visando equalizar possíveis problemas que a escala poderia apresentar. E por fim, a sexta etapa, na qual o Comitê Avaliador emitiu o último parecer sobre o processo de tradução transcultural do instrumento.

\section{Análise estatística}

Com o EME-BR já na língua portuguesa, utilizou-se a análise fatorial confirmatória e o teste de consistência interna ("Reability Test") para verificar a validade do mesmo.

A utilização dos modelos de análise fatorial confirmatória para confirmar a validade dos indicadores como agentes de seus respectivos constructos é realizada ajustando os dados de entrada, ou seja, a matriz de correlação amostral a um modelo de medida. Utilizando o LISREL $®$ versão 8.8 testou-se o modelo do EME-BR, obtendo-se estatísticas para avaliá-lo em termos absoluto. Com base na natureza ordinal dos dados do presente estudo e a não normalidade multivariada dos mesmos foi adotado o procedimento de estimação Mínimos Quadrados Ponderados Diagonalmente (Diagonally Weighed Least Squares - DWLS), a partir de matrizes de correlação policóricas e matrizes assintóticas de covariância (Flora \& CURRAN, 2004).

Como os modelos de análise fatorial confirmatória não possuem um único teste estatístico para avaliar a força do modelo construído, foram desenvolvidas várias medidas de adequação do ajuste que, quando forem usadas em conjunto, oferecem o suporte empírico para a validade de constructo. Os três tipos de medidas de ajuste geral, úteis na análise fatorial confirmatória, podem ser representadas por medidas de ajuste absoluto, incremental e parcimonioso. As medidas de ajuste absoluto determinam o grau em que o modelo de medida construído é capaz de predizer com o menor erro possível a matriz de variância-covariância ou a matriz de correlação utilizada na modelagem. Dentre as principais medidas de ajuste absoluto encontradas na literatura, pode-se citar o teste de $\mathrm{x}^{2}$ (Qui-Quadrado), o índice de qualidade do ajuste (GFI) e a raiz quadrada da média dos quadrados dos erros de aproximação (RMSEA), os quais foram utilizados para medir o grau com que o modelo global construído representa a matriz de entrada dos dados.

Medidas incrementais de ajuste são indicadores da qualidade do ajuste, que possibilitam comparar o modelo estimado com um modelo teórico nulo, ou seja, visa determinar se todos os indicadores estão associados a um único fator latente. As medidas incrementais de ajuste mais importantes, segundo Hair, Anderson, Tatham e Black (2005) são o índice de ajuste não-normalizado (NNFI), o índice de ajuste normalizado (NFI) e o índice de qualidade de ajuste calibrado (AGFI).

Um modelo é parcimonioso quando não contém coeficientes desnecessários, ou seja, é um modelo simples, mas com grande capacidade explicativa. As medidas de ajuste de parcimônia relacionam a bondade do modelo com o número de coeficientes necessários para alcançar esse nível de ajuste. No presente estudo, foi utilizado o índice de bondade de ajuste de parcimônia (PGFI).

No QUADRO 2, pode se visualizar os valores sugeridos como aceitáveis, de cada um dos parâmetros dessas três formas de medida de ajuste da análise fatorial confirmatória.

QUADRO 2 - Medidas para avaliar a qualidade de ajuste dos modelos estruturais.

\begin{tabular}{|c|c|}
\hline Medidas de qualidade de ajuste & Nivel de ajuste recomendado \\
\hline Medidas de ajuste absoluto & $\mathrm{p}>0,05$ \\
\hline$S-B x^{2}$ (Qui-Quadrado) & Superior a 0,90 \\
\hline GFI & Inferior a 0,08 \\
\hline RMSEA & \\
\hline Medidas de ajuste incremental & Superior a 0,90 \\
\hline NNFI & Superior a 0,90 \\
\hline NFI & Superior a 0,90 \\
\hline AGFI & \\
\hline Medidas de ajuste parcimonioso & \\
\hline PNFI & Sem valor definido. Valores maiores indicam maior parcimônia. \\
\hline
\end{tabular}


O teste de consistência interna, realizado no programa SPSS $®$ for Windows $®$, versão 15.0 por meio do índice de confiabilidade Alpha de Cronbach, tem como objetivo correlacionar os itens de cada escala de um grupo de respostas e, a partir desta

\section{Resultados}

\section{Análise fatorial confirmatória}

$\mathrm{Na}$ TABELA 1, pode-se avaliar o ajuste dos dados ao modelo proposto de sete fatores oblíquos e compará-lo com modelos alternativos.

Com exceção do teste $\mathrm{S}-\mathrm{Bx}^{2}$ que foi significante $(\mathrm{p}<$ 0,001 ), todas as outras medidas de ajuste absoluto (GFI e RMSEA) obtiveram valores dentro dos aceitáveis pela literatura (HAIR et al., 2005) no modelo hipotetizado.

Analisando-se os demais índices, observa-se que embora o modelo com um fator apresente uma qualidade de ajuste absoluto bastante similar ao modelo de seis fatores oblíquos, somente no último obtémse os valores do RMSEA dentro do limiar 0,08 correlação, chegar a um índice que varia entre 0 e 1. Netemeyer, Bearden e Sharma (2003) sugerem um índice Alpha de Cronbach acima de 0,70 como sendo preciso e confiável quanto a variável em que se pretende mensurar. sugerido como critério máximo aceitável (BROWNE \& Cudeck, 1993; Hair et al., 2005). Além disso, o modelo de sete fatores oblíquos revela índices adequados de ajuste incremental (NFI, NNFI e AGFI), excedendo o nível recomendado de 0,90 , e também o maior valor do índice parcimonioso (PNFI).

A correlação entre os fatores foi calculada por meio do teste de Spearman para examinar a validade discriminante do instrumento. Segundo BEM (2004), as correlações consideradas elevadas, geralmente acima de $\pm 0,90$, significam que tais fatores latentes não representam constructos diferentes, explicando informações redundantes. $\mathrm{Na}$ TABELA 2 pode-se observar as correlações entre os fatores da EME-BR.

TABELA 1 - Comparação de modelos confirmatórios alternativos para a EME-BR.

\begin{tabular}{lccccccccc}
\hline $\begin{array}{c}\text { Modelo } \\
\text { EME-BR }\end{array}$ & S-Bx & df & $\mathbf{p}$ & GFI & RMSEA (90\% IC) & AGFI & NFI & NNFI & PNFI \\
\hline $\begin{array}{l}7 \text { fatores } \\
\text { oblíquos }\end{array}$ & 687,49 & 329 & $\mathrm{p}<0,001$ & 0,969 & $0,054(0,049-0,060)$ & 0,962 & 0,945 & 0,966 & 0,823 \\
$\begin{array}{l}7 \text { fatores } \\
\text { ortogonais }\end{array}$ & 1770,92 & 350 & $\mathrm{p}<0,001$ & 0,463 & $0,105(0,100-0,110)$ & 0,377 & 0,859 & 0,874 & 0,795 \\
1 fator & 1464,89 & 350 & $\mathrm{p}<0,001$ & 0,937 & $0,093(0,088-0,098)$ & 0,927 & 0,883 & 0,901 & 0,818 \\
\hline
\end{tabular}

TABELA 2 - Correlações entre os fatores da EME-BR.

IC: Índice de Confiabilidade;

Nomes dos índices em inglês (HAIR et al., 2005): S-Bx² (Satorra-Bentler's chi-square test);

DF: degrees of freedom; GFI: Goodness of Fit Index;

RMSEA: Root Mean Square Error of Approximation;

AGFI: Adjusted Goodness of Fit Index;

NFI: Normed Fit Index; NNFI: Non-Normed Fit Index);

PNFI: Parsimony Normed Fit Index.

\begin{tabular}{lccccccc}
\hline & MI-C & MI-AO & MI-EE & ME-RE & ME-I & ME-ID & DES \\
\hline MI-C & 1,00 & & & & & & \\
MI-AO & $0,70^{* *}$ & 1,00 & & & & & \\
MI-EE & $0,63^{* *}$ & $0,61^{* *}$ & 1,00 & & & & \\
ME-RE & $0,47^{* *}$ & $0,44^{* *}$ & $0,32^{* *}$ & 1,00 & & & \\
ME-I & $0,53^{* *}$ & $0,50^{* *}$ & $0,45^{* *}$ & $0,52^{* *}$ & 1,00 & & \\
ME-ID & $0,55^{* *}$ & $0,50^{* *}$ & $0,41^{* *}$ & $0,57^{* *}$ & $0,57^{* *}$ & 1,00 & 1,00 \\
DES & $0,13^{* *}$ & $0,12^{*}$ & 0,05 & $0,32^{* *}$ & $0,21^{* *}$ & $0,26^{* *}$ & \\
\hline
\end{tabular}


A correlação mais forte ocorreu entre os três fatores de motivação intrínseca. Da mesma forma, os três fatores referentes a motivação extrínseca apresentaram uma correlação significativa.
Abaixo, pode-se visualizar o diagrama com as estimativas do modelo proposto, o qual descreve também a relação entre as dimensões e os indicadores na solução encontrada para os dados do EME-BR.

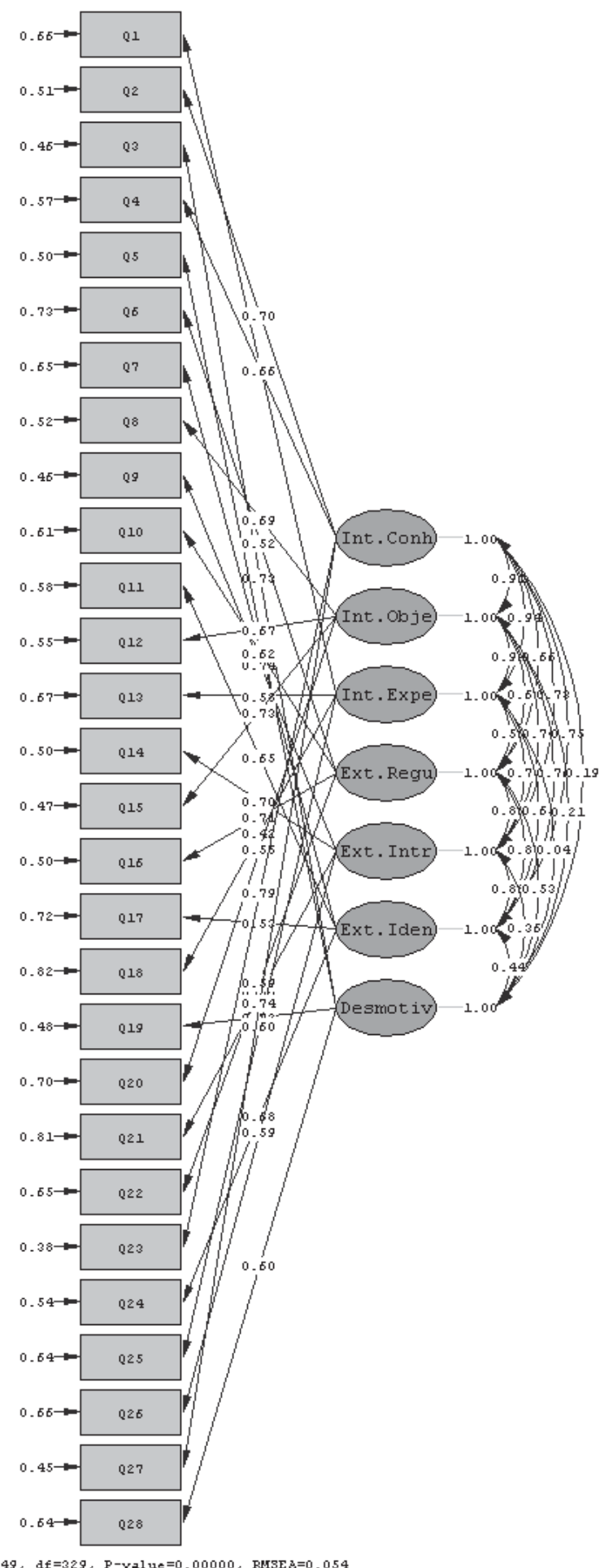

FIGURA 1- Estimativas do modelo de sete fatores oblíquos da EME-BR. 


\section{Confiabilidade interna}

Em termos de confiabilidade interna geral, os resultados apresentaram um índice Alpha de Cronbach de 0,90 para a EME-BR. Entretanto, como é uma escala multidimensional, torna-se mais importante calcular a confiabilidade de cada dimensão, de forma separada. Assim, a TABELA 3 apresenta os resultados da confiabilidade interna do presente estudo e de algumas pesquisas para cada uma das dimensões do EME-BR.
Como pode-se observar na TABELA 3, somente as dimensões de "Motivação intrínseca para conhecer" e "Motivação intrínseca para atingir objetivos" atingiram o valor mínimo $(>0,70)$ recomendado pela literatura (NetEMEyer, BEARden \& Sharma, 2003). Entretanto, as demais dimensões, excetuando-se a de "Motivação Intrínseca para experiências estimulantes" aproximam-se do índice desejável. Além disso, outras pesquisas tambem relatam índices de confiabilidade insuficientes do instrumento (ver TABELA 3).

TABELA 3 - Índicealpha de Cronbach para as dimensões do EME-BR no presente estudo eem diversas pesquisas.

\begin{tabular}{|c|c|c|c|c|c|c|c|}
\hline \multirow{2}{*}{ Pesquisas } & \multicolumn{7}{|c|}{ Índice alpha de Cronbach por dimensóes } \\
\hline & MI-C & MI-EE & MI-AO & ME-ID & ME-I & ME-RE & DES \\
\hline $\begin{array}{l}\text { PELLETIER et al. (1995) } \\
\text { Atletas universitários canadenses }\end{array}$ & 0,80 & 0,74 & 0,80 & 0,63 & 0,74 & 0,77 & 0,75 \\
\hline $\begin{array}{l}\text { LI \& HARMER (1996) } \\
\text { Atletas universitários masculinos }\end{array}$ & - & - & - & 0,68 & 0,79 & 0,79 & 0,80 \\
\hline $\begin{array}{l}\text { Li \& HARMER (1996) } \\
\text { Atletas universitários femininos }\end{array}$ & - & - & - & 0,70 & 0,74 & 0,77 & 0,82 \\
\hline $\begin{array}{l}\text { RiEMER, FinK \& FitZGERALD (2002) } \\
\text { Atletas adultos de "Curling" }\end{array}$ & 0,86 & 0,78 & 0,81 & 0,72 & 0,66 & 0,74 & 0,70 \\
\hline $\begin{array}{l}\text { Presente estudo } \\
\text { Atletas de futebol do Brasil }\end{array}$ & 0,76 & 0,57 & 0,71 & 0,67 & 0,67 & 0,65 & 0,62 \\
\hline
\end{tabular}

\section{Discussão}

O principal objetivo do presente estudo foi verificar as propriedades psicométricas da Escala de Motivação no Esporte na língua portuguesa brasileira com uma amostra de atletas jovens brasileiros de futebol de alto nível. Tanto a dimensionalidade quanto a confiabilidade da EME-BR foram investigados.

Interpretando os resultados encontrados na análise fatorial confirmatória obtém-se um maior suporte de validade do modelo proposto originalmente na versão inglesa da EME-BR. Apesar de o teste $\mathrm{S}-\mathrm{Bx}^{2}$ ter sido significante, indicando que o modelo proposto não apresenta um bom ajuste aos dados, esse teste pode falhar quando não há uma distribuição normal dos dados (SATORRA \& BENTLER, 1994; TOMARKEN \& WALLER, 2005), como é o caso do presente estudo. Além disso, diversos estudos identificaram o mesmo problema com esse índice de ajuste absoluto do modelo à estrutura fatorial proposta (LI \& HARMER, 1996; MALLETT et al., 2007; Martens \& Webber, 2002; Martin \& Cutler, 2002; Pelletier et al., 1995; Riemer,
Fink \& Fitzgerald, 2002; Shaw, Ostrow \& BeCKSTEAd, 2005; Standage, Duda \& NTOumanis, 2003). Com relação aos demais índices de ajuste absoluto, incremental e parcimonioso do modelo de sete fatores oblíquos, todos excederam os níveis recomendados, indicando uma boa dimensionalidade do instrumento na língua portuguesa brasileira.

Entre todos os constructos latentes foram encontradas correlaçôes positivas e em quase todos os casos significativas. Como já era de se esperar e também constatado em outras pesquisas (MALLETT et al., 2007; Martens \& Webber, 2002; Pelletier et al., 1995) as correlações mais fortes ocorreram entre as três dimensões de Motivação Intrínseca, já que essas estão relacionadas com um mesmo constructo (motivação intrínseca). Os valores obtidos para esses fatores no teste de correlação do presente estudo são muito similares a obtidas por PELLETIER et al. (1995) no estudo de validação do instrumento na língua inglesa, revelando que eles são semelhantes, mas não representam constructos idênticos. 
Além disso, espera-se que as correlações entre as sete dimensões do EME-BR representam a autodeterminação em um "continuum", conforme sugerido por DECI e RYAN (1985). Suporte para esse "continuum" é obtido por meio da correlação positiva entre fatores adjacentes (ex. Regulação Externa e Introjeção) e correlaçôes negativas ou quase nulas entre fatores opostos neste "continuum" (ex. Motivação Intrínseca e Desmotivação). Assim, encontra-se suporte na literatura (DECI \& RyAN, 1985; BRIÉRE et al., 1995) para os valores de correlação entre os fatores aqui encontrados.

Em relação aos resultados de consistência interna do EME-BR, somente duas dimensōes (MI-C e $\mathrm{MI}-\mathrm{AO}$ ) alcançaram níveis satisfatórios. Em outros estudos, também, foram relatados problemas de confiabilidade relacionados a todas as dimensões de motivação extrínseca: de Identificação (Li \& Harmer; 1996; Martin \& Cutler, 2002; PelleTiER et al., 1995; Vlachopoulos, KarageOrghis \& Terry, 2000), de Introjeção (Raedeke \& SMith, 2001; Riemer, Fink \& FitzGerald; 2002; StandaGe, Duda \& NToumanis, 2003) e para regulação externa (Raedeke \& Smith, 2001; Standage, Duda \& NTOUmanis, 2003). Apesar disso, os valores encontrados no presente estudo, para os respectivos fatores citados anteriormente estão bem próximo do nível desejado e alcançam, segundo Hair et al. (2005), o mínimo desejável para pesquisas exploratórias $(>0,60)$. Já o valor Alpha de Cronbach das dimensões "Motivação Intrínseca para experiências estimulantes" e "Desmotivação" foi bem inferior ao aceitável e o que foi relatado por outros estudos que analisaram as propriedades psicométricas da EME-BR na língua inglesa (Li \& HARMER; 1996; Pelletier et al., 1995). Dimensões que não apresentam uma alta confiabilidade $(\geq 0,70)$, de acordo com PASQUali (1999, 2007), podem mesmo assim ter seus dados analisados. Entretanto, o autor chama a atenção para o seguinte: sempre que alguma dimensão estiver abaixo desse nível de exigência, os pesquisadores e os leitores devem tomar cuidado na interpretação dos dados para não afirmarem categoricamente algumas conclusōes que podem estar inconsistentes devido a prováveis equívocos de compreensão do instrumento.

Portanto, pode-se dizer que embora o EME$\mathrm{BR}$ apresente alguns problemas relacionados principalmente a confiabilidade e a falta de ajuste absoluto adequado dos dados ao modelo proposto, os resultados obtidos no presente estudo dão algum suporte para a validade da EME-BR com atletas de futebol brasileiros jovens de alto rendimento.

De acordo com Pasquali (1999) e Marsh e JACKSON (1999), o processo de validação ecológica (validação externa) de um instrumento psicométrico pode durar anos e, às vezes, décadas, e não pode ser finalizado em somente um estudo, sendo assim, torna-se necessário o acompanhamento longitudinal do desempenho psicométrico deste instrumento na população de jovens futebolistas.

Assim, tendo em vista os problemas encontrados neste estudo relacionados com a especificação do modelo, recomenda-se a ampliação e a diversificação da amostra, para reduzir a probabilidade de se encontrarem problemas na $\mathrm{AFC}$ e relacionados a confiabilidade da EME-BR. Futuras pesquisas com o EME-BR devem buscar testar o modelo proposto entre outras variáveis de interesse como genero, esportes individuais e coletivos e em diferentes níveis competitivos. MARTEns e WebBER (2002) também recomendam testar a validade do instrumento em diferentes momentos da temporada (ex. preparação, competição, recuperação), podendo assim determinar se a estrutura fatorial da EME-BR permanece constante quando se varia esses fatores externos. Estudos revelam que o nível de motivação intrínseca, por exemplo, pode variar de acordo com a competição, o comportamento do treinador e experiências de fracasso e sucesso (para uma revisão ver Vallerand \& Losier, 1999).

Outra sugestão que deve ser considerada é a versão revisada do SMS (Sport Motivation Scale - SMS-6, vide MALLETT et al., 2007), a qual foi elaborada devido as limitações teóricas, empíricas e práticas da versao original do instrumento. O SMS6 inclui uma nova dimensão denominada "regulação integrada" e agrupa as três dimensões de motivação intrínseca em somente um fator com quatro itens. Embora Pelletier, Vallerand e Sarrazin (2007) tenham feito algumas críticas à versão revisada da SMS, a mesma apresenta propriedades psicométricas superiores às relatadas sobre a versão original. Mas conforme os próprios autores da SMS-6 afirmam, maiores estudos com a versão revisada em diferentes contextos e culturas são necessários, para que se possa avaliar qual versão é a mais adequada. Assim, recomenda-se a tradução da SMS-6 e o teste de suas propriedades psicométricas também sejam realizados na língua portuguesa brasileira. 


\begin{abstract}
Validation of sport motivation scale (SMS) in soccer for the Brazilian Portuguese language

The purpose of this study was to submit the Sport Motivation Scale (SMS) questionnaire for the transculture validation of the Sport Motivation Scale for the Brazilian Portuguese language and analysis the validity and applicability in the High level Brazilian younger football players. 370 athletes participated of this study. Was used the Sport Motivation Scale for the Brazilian Portuguese language questionnaire consisting for the 28 questions, in 7 dimensions. For the translation and adaptation of the instrument was used the conduct suggest in literature. The results of confirmatory factor analysis showed that with the exception of $S-B x^{2}$ that test was significant $(p<0.001)$, all other measures of absolute fit (GFI and RMSEA) and incremental fit (NFI, NNFI and AGFI), presented within the acceptable values in the literature. Regarding the completion of internal reliability, we can observe that only two dimensions have reached the minimum value $(>0.70)$, however, the other dimensions, closer to the desirable rate. However, the results showed in this study lend some support to the validity of the EME-BR with young Brazilian players to senior football.
\end{abstract}

UnITERMS: Motivation; High level football; Validation.

\title{
Referências
}

ALBUQUERQUE, M.R.; COSTA, V.T.; SAMULSKI, D.M.; NOCE, F. Avaliação do perfil motivacional dos atletas de alto rendimento do taekwondo brasileiro. Revista Iberoamericana de Psicología del Ejercicio y el Deporte, Las Palmas de Gran Canaria, v.3, n.1, p.74-94, 2008.

BEATON, D.E; BOMBARDIER,C.; GUILLEMIN, F. e FERRAZ, M.B. Guidelines for the process of cross-cultural adaptation of self-report measures. Spine, Philadelphia, v.25, n.24, p.3186-91, 2000.

BEM, A. Confiabilidade e validade estatísticas da avaliação docente pelo discente: proposta metodológica e estudo de caso. 2004. Tese (Doutorado em Engenharia de produção) - Universidade de Santa Catarina, Florianópolis, 2004.

BRIERE, N.; VALLERAND, R.; BLAIS, N.; PELLETIER, L. Développement et validation d'une mesure de motivation intrinsèque, extrinsèque et d'amotivation en contexte sportif : l'Échelle de motivation dans les sports (ÉMS). International Journal of Sport Psychology, Rome, v.26, p.465-89, 1995.

BROWNE, M.W.; CUDECK, R. Alternative ways of assessing model fit. In: BOLLEN, K.A.; LONG, J.S. (Eds.). Testing structural equation models. Newbury: Sage, 1993. p.136-62.

DECI, E.L.; RYAN, R.M. Intrinsic motivation and self-determinism in human behavior. New York: Plenum Press, 1985. . The "what" and "why" of goal pursuits: human needs and the self-determination of behaviour. Psychological Inquiry, Philadelphia, v.11, p.227-68, 2000.

DOSIL, J. Motivación motor en el deporte. In: DOSIL, J. Psicología de la actividad física y del deporte. 2. ed. Madrid: McGrawHill, 2008. p.139-66.

FLORA, D.; CURRAN, P. An empirical evaluation of alternative methods of estimation for confirmatory factor analysis with ordinal data. Psychological Methods, Washington, v.9, n.4, p.466-91, 2004.

FONSECA, A.M. A pscologia do deporto e a batalha da qualidade. Revista Portuguesa de Ciencias do Desporto, Porto, v.1, n.1, p.114-23, 2001.

HAIR, J.; ANDERSON, R.; TATHAM, R.; BLACK, W. Análise multivariada de dados. Porto Alegre: Bookman, 2005. LI, F.; HARMER, P. Testing the simplex assumption underlying the sport motivation scale: a structural equation modeling analysis. Research Quarterly for Exercise and Sport, Washington, v.67, n.4, p.395-405, 1996.

MALLETT, C.J.; KAWABATA, M.; NEWCOMBE, P. Progressing measurement in sport motivation with the SMS-6: a response to Pelletier, Vallerand, and Sarrazin. Psychology of Sport and Exercise, Amsterdam, v.8, n.5, p.622-31, 2007. MALlETT, C.J.; KAWABATA, M.; NEWCOMBE, P.; OTERO-FERERO, A.; JACKSON, S.A. Sports motivation Scale-6 (SMS-6): a revised six-factor sport motivation scale. Psychology of Sport and Exercise, Amsterdam, v.8, n.5, p.600-14, 2007. 
MARSH, H.W.; JACKSON, S.A. Flow experience in sport: construct validation of multidimensional hierarchical state and trait responses. Structural Equation Modeling, Philadelphia, v.6, 343-71, 1999.

MARTENS, M.P.; WEBBER, S.N. Psychometric properties of the sport motivation scale: an evaluation with College Varsity Athletes from the U.S. Journal of Sport \& Exercise Psychology, Champaign, v.24, p.254-70, 2002.

MARTIN, J.J.; CUTLER, K. An exploratory study of flow and motivation in theatre actors. Journal of Applied Sport Psychology, London, v.14, p.344-52, 2002.

NETEMEYER, R.; BEARDEN, W.; SHARMA, S. Scaling procedures: issues and applications. Thousand Oaks: Sage, 2003. PASQUALI, L. Instrumentos psicológicos: manual prático de elaboração. Brasília: Lab PAM, 1999.

Validade dos testes psicológicos: será possível reencontrar o caminho? Psicologia: Teoria e Pesquisa, Brasília, v.23, p.99-107, 2007.

PELLETIER, L.G.; SARRAZIN, P. Measurement issues in self-determination theory and sport. In: HAGGER, M.S.; CHATZISARANTIS, N. (Eds.). Self-determination theory in exercise and sport. Champaign: Human Kinetics, 2007. p.143-52. PELLETIER, L.G.; TUSON, D.M.; FORTIER, M.S.; VALLERAND, R.J.; BRIËRE, N.M.; BLAIS, M.R. Toward a new measure of intrinsic motivation, extrinsic motivation and amotivation in sports: the sport motivation scale. Journal of Sport \& Exercise Psychology, Champaign, v.17, n.1, p.35-53, 1995.

PELLETIER, L.G.; VALLERAND, R.J.; SARRAZIN, P. The revised six-factor Sport Motivation Scale (Mallett,Kawabata, Newcombe, Otero-Forero, \& Jackson, 2007): something old, something new, and something borrowed. Psychology of Sport and Exercise, Amsterdam, v.8, n.5, p.615-621, 2007.

RAEDEKE, T.D.; SMITH, A.L. Development and preliminary validation of an athlete burnout measure. Journal of Sport \& Exercise Psychology, Champaign, v.23, p.281-306, 2001.

REIMER, H.; FINK, J.S.; FITZGERALD, M.P. External validity of the Sport Motivation Scale. Avante, [S.l.],v.8, p.57-66, 2002.

RUIZ, E.J.F; BENEDICTO, L.V.; DOSIL, J.D. Nuevas aportaciones en psicología del deporte: una mirada crítica sobre la última década de la disciplina en Espana. Cuadernos de Psicología del Deporte, Muria, v.4, n.1/2, p.7-17, 2004.

RYAN, R.M; DECI, E.L. Self-determination theory and the facilitation of intrinsic motivation, social development, and well-being. American Psychologist, Washington, v.55, n.1, 2000.

SAMULSKI, D.M. Psicologia do esporte. Belo Horizonte: UFMG, 1995.

Motivação. In: SAMULSKI, D.M. Psicologia do esporte: conceitos e novas perspetivas. Barueri: Manole, 2009. p.167-92.

SATORRA, A.; BENTLER, P. Corrections to test statistics and standards errors in covariance structure analysis. In: EYE, A.; CLOGG, C. (Eds.). Latent variables analysis: applications for developmental research. Thousand Oaks: Sage, 1994. SHAW, K.L.; OSTROW, A.; BECKSTEAD, J. Motivation and the senior athlete: an examination of the psychometric properties of the Sport Motivation Scale. Topics in Geriatric Rehabilitation, Philadelphia, v.21, p.206-14, 2005.

STANDAGE, M.; DUDA, J.L.; NTOUMANIS, N. A model of contextual motivation in physical education: using constructs from self-determination and achievement goal theories to predict physical activity intentions. Journal of Educational Psychology, Baltimore, v.95, p.97-110, 2003.

TOMARKEN, A.; WALLER, N. Structural equation modeling as a data-analytic framework for clinical science: strengths, limitations, and misconceptions. Annual Review of Clinical Psychology, Palo Alto, v.1, p.31-65, 2005.

VALLERAND, R.J. Intrinsic and extrinsic motivation in sport and physical activity: a review and a look at the future. In: TENENBAUM, G.; EKLUND, E. (Eds.). Handbook of sport psychology. 3rd ed. New York: John Wiley, 2007. p.49-83. VALLERAND, R.J.; LOSIER, G.F. An integrative analysis of intrinsic and extrinsic motivation in sport. Journal of Applied Sport Psychology, London, v.11, p.142-69, 1999

VLACHOPOULOS, S.P.; KARAGEORGHIS, C.I.; TERRY, P.C. Motivation profiles in sport: a self-determination theory perspective. Research Quarterly for Exercise and Sport, Washington, v.71, p.387-97, 2000.

ENDEREÇO

Varley Teoldo da Costa Laboratório de Psicologia do Esporte

Escola de Educação Física, Fisioterapia e Terapia Ocupacional Universidade Federal de Minas Gerais

Av. Presidente Antônio Carlos, 6627 - Campus Pampulha 31270-901 - Belo Horizonte - MG - BRASIL e-mail: vtcosta@hotmail.com
Recebido para publicação: 07/ 12/ 2009

Revisado: 02/ 05/2011

Aceito: 13/07/2011 